\title{
Primeiro registro de Kroeberia fuliginosa Lindner, 1930 \\ (Diptera:Ropalomeridae) para o Estado do Pará, Brasil
}

\author{
Fernando da Silva Carvalho Filho ${ }^{1,3}$, Maria Cristina Esposito ${ }^{1}$ \& Rita de Cássia Oliveira dos Santos ${ }^{2}$ \\ Biota Neotropica $v 7$ (n3) \\ http://www.biotaneotropica.org.br/v7n3/pt/abstract?short-communication+bn04107032007 \\ Recebido em 27/08/07 \\ Versão Reformulada recebida em 31/10/07 \\ Publicado em 12/11/07 \\ ${ }^{I}$ Laboratório de Ecologia de Invertebrados, Departamento de Biologia, Universidade Federal do Pará, \\ Rua Augusto Corrêa, 01, CEP 66040-170, Guamá, Belém, PA, Brasil \\ ${ }^{2}$ Campus Universitário de Bragança, Universidade Federal do Pará \\ Av. Leandro Ribeiro, s/n, Aldeia, CEP 68600-000, Bragança, PA,Brasil \\ ${ }^{3}$ Autor para correspondência: Fernando da Silva Carvalho Filho, \\ e-mail:fernanbio@yahoo.com.br
}

\section{Abstract}

Carvalho Filho, F.S., Esposito M.C. \& Santos, R.C.O. First record of Kroeberia fuliginosa Lindner, 1930 (Diptera:Ropalomeridae) from Pará State, Brazil. Biota Neotrop. Sep/Dez 2007 vol. 7, no. 3 http://www. biotaneotropica.org.br/v7n3/pt/abstract?short-communication+bn04107032007. ISSN 1676-0603.

Kroeberia fuliginosa Lindner, 1930, was collected in the Pará state, Brazil, for the first time, extending the geographical distribution of this species.

Keywords: Kroeberia, Ropalomeridae, Diptera, Pará, new record.

\section{Resumo}

Carvalho Filho, F.S., Esposito M.C. \& Santos, R.C.O. Primeiro registro de Kroeberia fuliginosa Lindner, 1930 (Diptera:Ropalomeridae) para o Estado do Pará, Brasil. Biota Neotrop. Sep/Dez 2007 vol. 7, no. 3 http:// www.biotaneotropica.org.br/v7n3/pt/abstract?short-communication+bn04107032007. ISSN 1676-0603.

Kroeberia fuliginosa Lindner, 1930 foi coletada no estado do Pará pela primeira vez, ampliando assim a distribuição geográfica dessa espécie.

Palavras-chave: Kroeberia, Ropalomeridae, Diptera, Pará, novo registro. 


\section{Introdução}

Ropalomeridae são moscas robustas com 6-12 mm, olhos salientes, face com carena mediana ou tubérculo central, palpos alargados, fêmures alargados com cerdas ventrais fortes, tíbia posterior geralmente achatada lateralmente e encurvada (Alves \& Ale-Rocha 2006). A família inclui oito gêneros e 35 espécies (Alves \& Ale-Rocha 2006). É principalmente neotropical, ocorrendo desde o Sul dos Estados Unidos da América até o Norte da Argentina. Uma única espécie é conhecida da Região Neártica, Rhytidops floridensis (Aldrich,1932). Para o estado do Pará foram registrados até o presente, três gêneros e sete espécies (Prado \& Papavero 2002, Marques \& Ale-Rocha 2004, Marques \& Ale-Rocha 2005).

Kroeberia Lindner, 1930 é um gênero de moscas castanhas, de tamanho médio, com fronte pouco côncava, face com tubérculo central arredondado, arista nua ou com pêlos microscópicos, escutelo cerdoso e pernas com os fêmures bastante engrossados (Marques \& Ale-Rocha 2004). O gênero foi revisto por Marques \& Ale-Rocha (2004) e compreende duas espécies Kroeberia fuliginosa Lindner, 1930 e Kroeberia minor Marques \& Ale-Rocha, 2004. K. fuliginosa apresenta ampla distribuição podendo ser encontrada do México ao sul do Brasil (Steyskal 1967, Marques \& Ale-Rocha 2004) e K. minor ocorre somente no Brasil. Nesse artigo, a distribuição geográfica de Kroeberia fuliginosa é ampliada.

\section{Material e Métodos}

O espécime analisado foi coletado na Mata do Lobão, Município de Bragança, Pará, Brasil, em junho de 2007, por R. C. Santos utilizando-se armadilha para a captura de borboletas, como descrita por Rydon (1964), com banana fermentada como isca. A Mata do Lobão é uma floresta secundária com $1 \mathrm{~km}^{2}$ localizada próxima ao rio Caeté ( $1^{\circ} 02^{\prime}$ ' $04^{\prime \prime} \mathrm{S}$ e $\left.46^{\circ} 45^{\prime} 48^{\prime \prime} \mathrm{W}\right)$. O material foi preservado em álcool $70 \%$ e posteriormente montado em alfinetes.

O espécime analisado foi depositado na Coleção Entomológica do Museu Paraense Emílio Goeldi (MPEG) e identificado utilizando-se a chave de Alves \& Ale-Rocha (2006).

\section{Resultados e Discussão}

A ocorrência de Kroeberia fuliginosa Lindner, 1930 é assinalada, pela primeira vez, para o estado do Pará.

\section{Kroeberia fuliginosa Lindner, 1930}

Kroeberia fuliginosa Lindner, 1930:127; Steyskal, 1967:1 (catálogo); Prado \& Papavero, 2002: 1 (catálogo); Marques \& Ale-Rocha, 2004: 316 (redescrição, distribuição geográfica).

Diagnose: face com tubérculo central arredondado; cerdas orbitais ausentes; ocelares, pós-ocelares, vertical interna e externa presentes; arista nua; escutelo revestido por cerdas espiniformes com 1 cerda apical e 2 discais.

Material examinado: 1 macho: BRASIL, Pará, Bragança, 11.VI.2007, Santos, R. C. col.

\section{Lista de Espécies para o Estado do Pará}

Segue abaixo a lista, em ordem alfabética, das espécies de Ropalomeridae que ocorrem no estado do Pará, Brasil, mencionando a distribuição das espécies (Steyskal 1967, Prado \& Papavero 2002, Marques \& Ale-Rocha 2004, Marques \& Ale-Rocha 2005).

\section{Kroeberia fuliginosa Lindner, 1930}

Distribuição: México, Panamá, Venezuela, Brasil (Acre, Amapá, Roraima, Amazonas, Pará, Mato Grosso, Goiás, Rio de Janeiro, São Paulo e Rio Grande do Sul).

\section{Kroeberia minor Marques \& Ale-Rocha, 2004}

Distribuição: Brasil (Pará e Amazonas).

\section{Ropalomera tesselata Prado, 1966}

Distribuição: Brasil (Pará, Amapá, Amazonas).

\section{Ropalomera tibialis Walker, 1852}

Distribuição: Brasil (Pará, Amapá, Amazonas).

\section{Ropalomera titillator Steyskal, 1967}

Distribuição: Brasil (Pará)

\section{Willistoniella pleuropunctata (Wiedemann, 1824)}

Distribuição: México, Belize, Guatemala, Honduras, Curaçao, Costa Rica, Panamá, Trinidade, Suriname, Guiana, Venezuela, Colômbia, Equador, Peru, Brasil (Roraima, Pará, Amazonas, Tocantis, Rondônia, Sergipe, Maranhão, Bahia, Mato Grosso, Distrito Federal, Goiás, Espírito Santo, Rio de Janeiro, Minas Gerais, São Paulo e Santa Catarina), Bolívia, Paraguai e Argentina.

\section{Willistoniella spatulata Marques \& Ale-Rocha, 2005}

Distribuição: Honduras, Panamá, Venezuela, Colômbia, Equador e Brasil (Pará e Amazonas).

\section{Willistoniella ulyssesi Marques \& Ale-Rocha, 2005}

Distribuição: México, Guatemala, Belize, Honduras, Costa Rica, Panamá, Trinidade, Venezuela, Equador, Peru, Bolívia, Paraguai, Brasil (Acre, Roraima, Amapá, Pará, Amazonas, Rondônia, Goiás, Espírito Santo, Rio de Janeiro e Paraná).

\section{Conclusão}

Kroeberia fuliginosa é acrescida à lista de espécies que ocorrem no Pará, aumentando assim o conhecimento sobre a diversidade dos Ropalomeridae nesse estado.

O registro de Kroeberia fuliginosa no Pará amplia distribuição geográfica da espécie.

\section{Agradecimentos}

Os autores agradecem a Ivanei Araújo por ceder as armadilhas para borboletas utilizadas na captura das moscas e Marielda Góis, Ana Cristina Monteiro e o Sr. Porisso pelo auxílio no trabalho de campo. Aos dois revisores anônimos pelas sugestões propostas.

\section{Referências Bibliográficas}

ALVES, V.R. \& ALE-ROCHA, R. 2006. Novos registros e espécies de Ropalomeridae (Insecta, Diptera) do Mato Grosso, Brasil. In XXI Congresso Brasileiro de Entomologia (R. Barros, coord.). Recife, ID:1045-2.

ALDRICH, J. M. 1932. New Diptera, or two-winged flies from America, Asia, and Java, with additional notes. Proc. U. S. Natl. Mus. 81:1-28.

LINDNER, E. 1930. Revision der amerikanischen Dipteren-Familie der Rhopalomeridae. Dtsche. Entomol. Z. 2:122-137.

MARQUES, A.P.C. \& ALE-ROCHA, R. 2004. Revisão do gênero Kroeberia Lindner (Diptera, Ropalomeridae) da Região Neotropical. Rev. Bras. Entomol. 48:315-322.

MARQUES, A.P.C. \& ALE-ROCHA, R. 2005. Revisão do gênero Willistoniella Mik, 1985 (Díptera,Ropalomeridae) da Região Neotropical. Rev. Bras. Entomol. 49:210-227

PRADO, A. P. \& PAPAVERO, N. 2002. Insecta - Diptera - Ropalomeridae. In: Fauna da Amazônia Brasileira (N. PAPAVERO, ed.). Belém, Museu Paraense Emílio Goeldi, v. 5, p. 1-3.

RYDON, A. 1964. Notes on the use of butterfly traps in East Africa. Journal of the Lepidopterists' Society, 18:51-58.

STEYSKAL, G.C. 1967. Family Ropalomeridae. In: A catalogue of the Diptera of the Americas South of the United States (N. Papavero ed.). São Paulo, Departamento de Zoologia, Secretaria da Agricultura, v. 60, p. 1-7. 